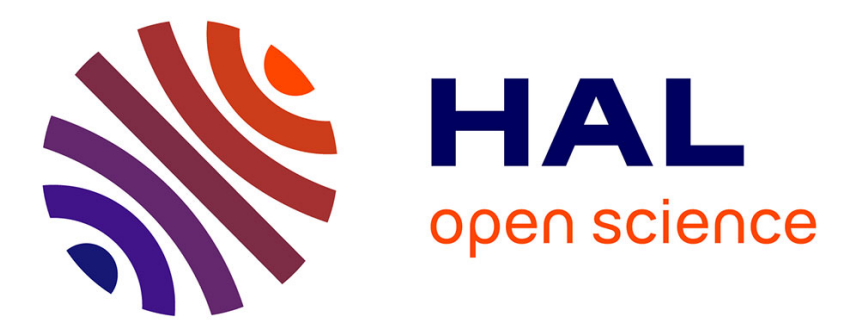

\title{
Influence of heat transfer on the estimation of water vapor diffusion coefficient in transient regime
}

\author{
Yves Jannot, Harouna Mamadou Bal, Alain Degiovanni, Christian Moyne
}

\section{To cite this version:}

Yves Jannot, Harouna Mamadou Bal, Alain Degiovanni, Christian Moyne. Influence of heat transfer on the estimation of water vapor diffusion coefficient in transient regime. International Journal of Heat and Mass Transfer, 2021, 177, pp.121558. 10.1016/j.ijheatmasstransfer.2021.121558 . hal-03265547

\section{HAL Id: hal-03265547 \\ https://hal.univ-lorraine.fr/hal-03265547}

Submitted on 21 Jun 2021

HAL is a multi-disciplinary open access archive for the deposit and dissemination of scientific research documents, whether they are published or not. The documents may come from teaching and research institutions in France or abroad, or from public or private research centers.
L'archive ouverte pluridisciplinaire HAL, est destinée au dépôt et à la diffusion de documents scientifiques de niveau recherche, publiés ou non, émanant des établissements d'enseignement et de recherche français ou étrangers, des laboratoires publics ou privés.

\section{(1) (1) $\$$}

Distributed under a Creative Commons Attribution - NonCommercial - NoDerivatives| 4.0 


\title{
Influence of heat transfer on the estimation of water vapor diffusion coefficient in transient regime ${ }^{1}$
}

\author{
Yves Jannot $^{1}$, Harouna Mamadou Bal ${ }^{2}$, Alain Degiovanni ${ }^{1,3}$, Christian Moyne $^{1}$ \\ ${ }^{1}$ Université de Lorraine, CNRS, LEMTA, 54000 Nancy, France \\ ${ }^{2}$ LM3E, IUT de l'Université de Thiès, Thiès, Sénégal \\ ${ }^{3}$ Université Internationale de Rabat, Pôle Energie, LERMA, Rocade Rabat-Salé, Sala Al Jadida, 11100, \\ Morocco
}

\begin{abstract}
The use of bio-sourced materials for thermal insulation of buildings is growing mainly for environmental reasons. It has already been demonstrated that since these materials are highly hydrophilic, mass transfer may have significant influence on their temperature field. We first develop a heat and mass transfer model with a limited number of parameters and we use it to simulate the mass and temperature fields during a typical transient experiment dedicated to vapor diffusion coefficient estimation. Estimations made from these simulations with a simple mass transfer model show that the estimation error can be greater than $30 \%$ in some cases. A sensitivity study shows that the vapor diffusion coefficient, the thermal conductivity and the external mass transfer coefficient can be estimated separately from a mass recording, with the coupled model. An experimental study carried out at $15{ }^{\circ} \mathrm{C}, 25{ }^{\circ} \mathrm{C}$ and $35^{\circ} \mathrm{C}$ on a wood fiber board confirms the results of the theoretical study. The deviation between the vapor diffusion coefficient estimated with and without taking into account the heat transfer was $13.3 \%$ at 35 ${ }^{\circ} \mathrm{C}$. It is finally shown that the isosteric heat of sorption has no effect on the estimation of the diffusion coefficient.
\end{abstract}

Keywords : bio-sourced material, insulating material, heat transfer, mass transfer, vapor diffusion coefficient

Nomenclature

$c_{w} \quad$ Specific heat of water $\left(\mathrm{J} \mathrm{kg}^{-1} \mathrm{~K}^{-1}\right)$

$c_{S} \quad$ Specific heat of the solid in dry state $\left(\mathrm{J} \mathrm{kg}^{-1} \mathrm{~K}^{-1}\right)$

$D \quad$ Mass diffusivity $\left(\mathrm{m}^{2} \mathrm{~s}^{-1}\right)$

$D_{X}^{l} \quad$ Isothermal mass diffusivity in liquid phase $\left(\mathrm{m}^{2} \mathrm{~s}^{-1}\right)$

$D_{T}^{l} \quad$ Non-isothermal mass diffusivity in liquid phase $\left(\mathrm{m}^{2} \mathrm{~s}^{-1} \mathrm{~K}^{-1}\right)$

$D_{X}^{v} \quad$ Isothermal mass diffusivity in vapor phase $\left(\mathrm{m}^{2} \mathrm{~s}^{-1}\right)$

$D_{T}^{v} \quad$ Non-isothermal mass diffusivity in vapor phase $\left(\mathrm{m}^{2} \mathrm{~s}^{-1} \mathrm{~K}^{-1}\right)$

$D^{*} \quad$ Diffusivity of the water vapor in air $\left(\mathrm{m}^{2} \mathrm{~s}^{-1}\right)$

$e \quad$ Sample thickness (m)

$h_{c} \quad$ Convection heat transfer coefficient $\left(\mathrm{W} \mathrm{m}^{-2} \mathrm{~K}^{-1}\right)$

$h_{m} \quad$ External mass transfer coefficient $\left(\mathrm{kg} \mathrm{m}^{-2} \mathrm{~s}^{-1}\right)$

$h_{v} \quad$ External mass transfer coefficient $\left(\mathrm{m} \mathrm{s}^{-1}\right)$

$h_{r} \quad$ Radiation heat transfer coefficient $\left(\mathrm{W} \mathrm{m}^{-2} \mathrm{~K}^{-1}\right)$

$H_{l} \quad$ Enthalpy of the liquid $\left(\mathrm{J} \mathrm{kg}^{-1}\right)$

$H_{v} \quad$ Enthalpy of the vapor $\left(\mathrm{J} \mathrm{kg}^{-1}\right)$

${ }^{1}$ International Journal of Heat and Mass Transfer 177(2021) 121558 https://doi.org/10.1016/j.ijheatmasstransfer.2021.121558 
$H R \quad$ Relative humidity of air

$L \quad$ latent heat of vaporization $\left(\mathrm{J} \mathrm{kg}^{-1}\right)$

$m \quad$ Mass of the sample $(\mathrm{kg})$

$m_{s} \quad$ Dry mass of the sample $(\mathrm{kg})$

$\vec{m}_{l} \quad$ Liquid mass flow rate $\left(\mathrm{kg} \mathrm{m}^{-2} \mathrm{~s}^{-1}\right)$

$\vec{m}_{v} \quad$ Vapor mass flow rate $\left(\mathrm{kg} \mathrm{m}^{-2} \mathrm{~s}^{-1}\right)$

$M_{v} \quad$ Molar mass of air $(\mathrm{kg})$

$N \quad$ Number of experimental points

$p_{\text {sat }} \quad$ Pressure of saturated vapor at the temperature $T(\mathrm{~Pa})$

$p \quad$ Total gas pressure $(\mathrm{Pa})$

$p_{v} \quad$ Partial pressure of water vapor $(\mathrm{Pa})$

$T \quad$ Temperature $(\mathrm{K})$

$x \quad$ Distance from the air/sample interface (m)

$x_{v} \quad$ Mass fraction of water vapor in air $\left(\mathrm{kg} \mathrm{kg}_{\text {dry air }}{ }^{-1}\right)$

$X \quad$ Water content of the sample $\left(\mathrm{kg} \mathrm{kg}_{\text {dry mass }}{ }^{-1}\right)$

$X_{v} \quad$ Water vapor content of the sample $\left(\mathrm{kg} \mathrm{kg}_{\text {dry mass }}{ }^{-1}\right)$

$X_{l} \quad$ Water liquid content of the sample $\left(\mathrm{kg} \mathrm{kg}_{\text {dry mass }}{ }^{-1}\right)$

$X_{0} \quad$ Initial water content of the sample $\left(\mathrm{kg} \mathrm{kg}_{\text {dry mass }}{ }^{-1}\right)$

$X_{1} \quad$ Water content of the sample in equilibrium with the air flow ( $\mathrm{kg} \mathrm{kg}_{\text {dry mass }}{ }^{-1}$ )

$\bar{X} \quad$ Mean water content of the sample $\left(\operatorname{kg~kg}_{\text {dry mass }}{ }^{-1}\right)$

$Y_{1} \quad$ Air absolute humidity far from the surface $\left(\mathrm{kg} \mathrm{m}^{-3}\right)$

$Y_{0} \quad$ Air absolute humidity far on the surface $\left(\mathrm{kg} \mathrm{m}^{-3}\right)$

Greek symbols

$\delta \quad$ Thermomigration coefficient

$\Delta H_{s} \quad$ Isosteric heat of sorption $\left(\mathrm{J} \mathrm{kg}^{-1}\right)$

$\varepsilon_{g} \quad$ Volume fraction of the gaseous phase

$\varepsilon_{s} \quad$ Volume fraction of the solid phase

$\vec{\varphi} \quad$ Enthalpy flow rate $\left(\mathrm{J} \mathrm{m}^{-2} \mathrm{~s}^{-1}\right)$

$\lambda \quad$ Thermal conductivity at local scale $\left(\mathrm{W} \mathrm{m}^{-1} \mathrm{~K}^{-1}\right)$

$\lambda_{s} \quad$ Thermal conductivity of the (wet) solid phase $\left(\mathrm{W} \mathrm{m}^{-1} \mathrm{~K}^{-1}\right)$

$\lambda_{g}$ Thermal conductivity of the gaseous phase $\left(\mathrm{W} \mathrm{m}^{-1} \mathrm{~K}^{-1}\right)$

$\rho_{l} \quad$ Liquid density $\left(\mathrm{kg} \mathrm{m}^{-3}\right)$

$\rho_{v} \quad$ Vapor density $\left(\mathrm{kg} \mathrm{m}^{-3}\right)$

$\rho_{0} \quad$ Dry medium density $\left(\mathrm{kg} \mathrm{m}^{-3}\right)$

$\tau \quad$ Coefficient of tortuosity

\section{Introduction}

The use of bio-based insulating materials is currently developing because they allow limiting the emission of greenhouse gases by reducing the energy consumption linked to the heating of buildings while having environmental advantages: they require low consumption of energy for their manufacture and do not generate toxic emissions or waste. Their thermal conductivity is however slightly higher than that of insulating materials derived from petroleum products such as polyurethane foam for example. Among the main bio-based insulating materials that have been the subject of recent studies, we can cite hemp [15,18], wool [21], materials based on wood fiber [7,14]. 
They are also hygrophilic with water contents showing significant variations with the relative humidity of the ambient air: for example, the water content of pine varies from $5 \%$ to $15 \%$ when the humidity of the air varies from $20 \%$ to $60 \%$ [20]. When subjected to variations in air humidity, there is a water vapor mass transfer which has been shown to cause temperature variations of several degrees [19]. To predict the thermal behavior of a wall comprising this type of material, it is necessary to solve the problem of coupled heat and mass transfer and therefore to know the mass diffusion coefficient of water vapor in the material.

One of the most popular methods is the cup method which is a widely used steady state method $[4,6]$. This method involves subjecting the sample to be tested to a relative humidity gradient. The sample is sealed in a cup containing a saturated saline solution which imposes a constant and known relative humidity $H R_{0}$. The dish is placed in an environment with relative humidity $H R_{1}$ which is also constant and known, for example in an enclosure regulated in temperature and humidity. The mass of the cup is measured until it varies linearly, allowing the constant water vapor flow rate through the sample to be calculated and then the mass diffusion coefficient of the sample to be deduced. However, the measurement is very long and does not allow the estimation of the resistance to external transfer, like all steady-state measurements. These resistances may not be negligible for materials with high mass diffusivity [22] such as low density insulating materials.

Transient regime methods have also been used by many authors, they are all based on a common principle: a sample, which is at a uniform temperature and water content, is subjected to a sudden jump in the relative humidity of the air with which it is in contact and the evolution of its mass is recorded from this moment, as represented in Figure 1. We then estimate the unknown parameters (including the mass diffusivity) by minimizing the sum of the quadratic differences between the experimental points and a model representing the evolution of mass.

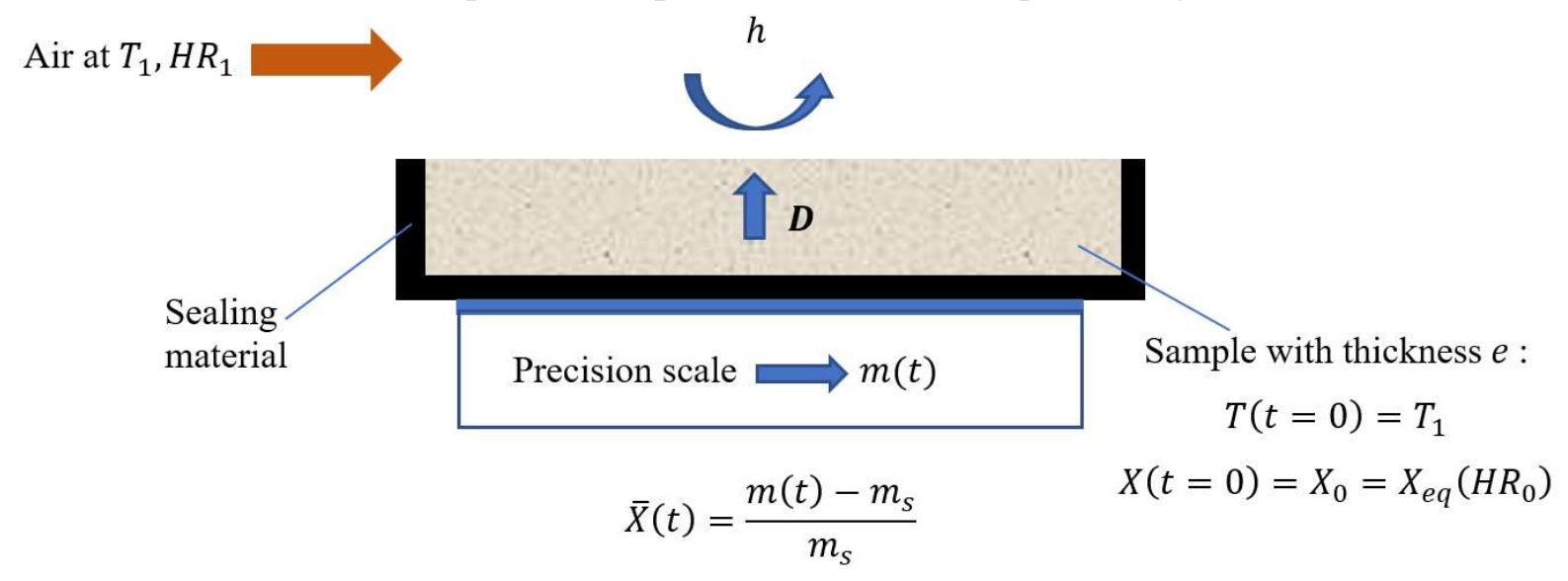

Figure 1: Classical experimental device for the measurement of the water vapor diffusion coefficient in transient regime

The methods may differ by the experimental device used, some authors use a DVS (Dynamic Vapor Sorption) device which allows to work on very small samples but which requires that the material is perfectly homogeneous [23], others use larger samples placed in an enclosure regulated in temperature and humidity [1]. A variant of this family of methods has recently been proposed in which the measurement of mass is replaced by a measurement of relative humidity on the sealed face of the sample [3].

The models used to represent the evolution of the mass of the sample generally do not take into account the heat transfer coupled with the mass transfer [5,23]. A theoretical study carried out 
starting from the numerical resolution of a model of coupled heat and mass transfer showed that the estimation error could be of $10 \%$ with a model taking into account the resistances to the external mass transfer and of $20 \%$ for a model not taking them into account [1]. However, this study was carried out considering solid wood with a lower mass diffusivity and higher thermal conductivity than those of a wood fiber panel for example.

We propose in this article a coupled heat and mass transfer model as well as a numerical method of resolution. This model will be used first to assess the error made when estimating the mass diffusion coefficient with models that do not take heat transfer into account. This study, carried out on ranges of thermal conductivity and mass diffusion coefficient wider than those considered by [1], will show that the errors can exceed $30 \%$ for insulating materials of high permeability such as wood fiber board.

This model will then be used to carry out a study of the reduced sensitivities of the mass of the sample (the measured quantity) to the unknown parameters that one seeks to estimate, namely the mass diffusion coefficient, the thermal conductivity and the coefficient of external mass transfer. It will allow to conclude that the unknown parameters can be estimated separately.

The results of an experimental study carried out on a wood fiberboard using a device of the type shown in Figure 1 at three different temperatures will then be presented. Estimates made using two mass transfer models and the coupled heat and mass transfer model will confirm the results of the theoretical study, with deviations in between the estimated values reaching $32 \%$ in the worst case. The temperature measurement carried out at the center of the sample will highlight temperature variations that can reach $5{ }^{\circ} \mathrm{C}$, thus confirming the results of [19] and showing that mass transfer must be taken into account to calculate the evolution of the temperature field in this type of material. The influence of the isosteric heat of sorption neglected in the model will finally been studied.

\section{Model and estimation methods}

\section{Model}

We will first present the coupled heat and mass transfer model used for this study, corresponding to the configuration of the system shown in Figure 1. In this study, it is considered that the gaseous pressure inside the sample is uniform and constant.

The enthalpy transferred to a humid material under the effect of a temperature and humidity gradient can be written [17]:

$\vec{\varphi}=-\lambda \overrightarrow{\nabla T}+\vec{m}_{l} H_{l}+\vec{m}_{v} H_{v}$

With:

$\lambda \quad$ Thermal conductivity

$T \quad$ Temperature

$\vec{m}_{l} \quad$ Liquid mass flow rate

$H_{l} \quad$ Enthalpy of the liquid

$\vec{m}_{v} \quad$ Vapor mass flow rate

$H_{v} \quad$ Enthalpy of the vapor

Where:

$\vec{m}_{l}=-\rho_{l}\left(D_{X}^{l} \overrightarrow{\nabla X_{l}}+D_{T}^{l} \overrightarrow{\nabla T_{l}}\right)$

$\vec{m}_{v}=-\rho_{v}\left(D_{X}^{v} \overrightarrow{\nabla X_{v}}+D_{T}^{v} \overrightarrow{\nabla T_{l}}\right)$

With:

$X_{v} \quad$ Water vapor content 
$X_{l} \quad$ Water liquid content

$\rho_{l} \quad$ Liquid density

$\rho_{v} \quad$ Vapor density

$D_{X}^{l} \quad$ Isothermal mass diffusivity $\left(\mathrm{m}^{2} \mathrm{~s}^{-1}\right)$ in liquid phase

$D_{T}^{v} \quad$ Non-isothermal mass diffusivity $\left(\mathrm{m}^{2} \mathrm{~s}^{-1}\right)$ in liquid phase

$D_{X}^{v} \quad$ Isothermal mass diffusivity $\left(\mathrm{m}^{2} \mathrm{~s}^{-1}\right)$ in vapor phase

$D_{T}^{v} \quad$ Non-isothermal mass diffusivity $\left(\mathrm{m}^{2} \mathrm{~s}^{-1}\right)$ in vapor phase

A mass balance carried out on an elementary volume of liquid and then of vapor allows us to write [17]:

$\rho_{0} \frac{\partial X_{l}}{\partial t}=\frac{\partial}{\partial x}\left[\rho_{0}\left(D_{X}^{l} \frac{\partial X_{l}}{\partial x}+D_{T}^{l} \frac{\partial T}{\partial x}\right)\right]-\dot{m}$

$\rho_{0} \frac{\partial X_{v}}{\partial t}=\frac{\partial}{\partial x}\left[\rho_{0}\left(D_{X}^{v} \frac{\partial X_{v}}{\partial x}+D_{T}^{v} \frac{\partial T}{\partial x}\right)\right]+\dot{m}$

Where $\dot{m}$ is the quantity of water evaporated per volume unit of porous medium and per unit of time, and $\rho_{0}$ is the density of the dry medium.

By adding the two relations, we get:

$\rho_{0} \frac{\partial X}{\partial t}=\frac{\partial}{\partial x}\left[\rho_{0}\left(D_{X} \frac{\partial X}{\partial x}+D_{T} \frac{\partial T}{\partial x}\right)\right]$

Where: $\quad X=X_{v}+X_{l}$

$$
\begin{aligned}
& D_{T}=D_{T}^{v}+D_{T}^{l} \\
& D_{X}=D_{X}^{v}+D_{X}^{l}
\end{aligned}
$$

By neglecting the accumulation term compared to the transport term in the vapor mass balance, we can write:

$\dot{m}=-\frac{\partial}{\partial x}\left[\rho_{0}\left(D_{X}^{v} \frac{\partial X}{\partial x}+D_{T}^{v} \frac{\partial T}{\partial x}\right)\right]$

A thermal balance carried out on an elementary volume of the wet porous medium gives:

$\rho_{0} c^{*} \frac{\partial T}{\partial t}=\frac{\partial}{\partial x}\left[\lambda^{*} \frac{\partial T}{\partial x}+\rho_{0} D_{X}^{v} L \frac{\partial X}{\partial x}\right]$

Where $\lambda^{*}$ is the apparent thermal conductivity.

It may be expressed by [2]:

$\lambda^{*}=\lambda+\rho_{0} D_{T}^{v} L$

Where:

Assuming that the isosteric heat of sorption is negligible (this hypothesis will be further discussed), $L$ is the vaporization latent heat of water given by [10]:

$L=2.501 \times 10^{6}-2650(T-273.15)$

$c^{*}=c_{s}+(1+X) c_{w}$

$c_{w} \quad$ specific heat of water

$c_{s} \quad$ specific heat of the solid in dry state

The parameter $\lambda$ in relation (9) represents the thermal conductivity at the local scale. In the general case, this conductivity depends on the thermal conductivities of the different phases (solid and gas) but also on the transport of vapor at the microscopic scale [2]. In the particular case of a parallel model (all the constituents of the material are in parallel with respect to the heat flow), it may be written [16]:

$\lambda=\varepsilon_{s} \lambda_{s}+\varepsilon_{g} \lambda_{g}$

In the following applications, the parameter $\lambda$, supposed constant, will be identified.

The diffusion coefficients of water vapor in a medium are written [17]:

$D_{X}^{v}=\frac{\tau}{\rho_{0}} \frac{D^{*}}{R T} \frac{M_{v}}{1-x_{v}} p_{\text {sat }} \frac{d H R}{d X}$ 
$D_{T}^{v}=\frac{\tau}{\rho_{0}} \frac{D^{*}}{R T} \frac{M_{v}}{1-x_{v}} H R \frac{d p_{s a t}}{d T}$

According to [7]: $D^{*}=2.17 \times 10^{-5}\left(\frac{T}{273}\right)^{1,88}$

According to [10] : $x_{v}=\frac{p_{v}}{p}$

Where $p_{v}$ is the partial pressure of water vapor) $\left(p_{v}=H R p_{\text {sat }}\right)$

And: $p_{\text {sat }}=\exp \left(25.5-\frac{5195}{T}\right)$

This relation is valid for $0{ }^{\circ} \mathrm{C}<T<60^{\circ} \mathrm{C}$, with $p_{\text {sat }}$ in $\mathrm{Pa}$ and $T$ in $\mathrm{K}$.

2.2 Solutions of the diffusion equation

For the sake of simplification of the writings, we will further note $D=D_{X}$ and $\delta=\frac{D_{T}}{D_{X}}$

Case where $D$ is constant and the heat transfer is negligible

If we can consider the mass diffusivity constant (small variation interval of $X$ ) and if we neglect the influence of heat transfer on mass transfer, the system of equations to be solved is as follows:

$\frac{\partial^{2} X}{\partial x^{2}}=\frac{1}{D} \frac{\partial X}{\partial t}$

With the initial condition:

$X(x, t=0)=X_{0}$

The first boundary condition at the air/product interface is :

at $x=0: h_{m}\left[X(x=0)-X_{1}\right]=-\rho_{0} D\left(\frac{\partial X}{\partial x}\right)_{x=0}$

Model M1: semi-infinite medium, $D$ constant, heat transfer and external resistance neglected

A first solution, which we will call the M1 model, can be developed by assuming that the sample is a semi-infinite medium:

$X(x, t)=X_{0}$ when $x \rightarrow \infty$

Neglecting the external mass transfer resistance, relation (19) becomes: $X(x=0, t)=X_{1}$

The problem is analogous to heat transfer in a semi-infinite medium with imposed surface temperature and the solution is written [12]:

$\frac{X(x, t)-X_{1}}{X_{0}-X_{1}}=\operatorname{erfc}\left(\frac{x}{2 \sqrt{D t}}\right)$

The mean value in the sample is then calculated between $x=0$ and $x=e$ :

$\bar{X}(t)=\frac{1}{e} \int_{0}^{e} X(x, t) d x=\frac{1}{e} \int_{0}^{e}\left[X_{1}+\left(X_{0}-X_{1}\right) \operatorname{erfc}\left(\frac{x}{2 \sqrt{D t}}\right)\right] d x$

With:

$\int \operatorname{erfc}(k x) d x=x \operatorname{erfc}(k x)-\frac{\exp \left(-k^{2} x^{2}\right)}{k \sqrt{\pi}}$

$\bar{X}(t)=X_{1}+\left(X_{0}-X_{1}\right) \frac{1}{e}\left[\operatorname{eerfc}\left(\frac{e}{2 \sqrt{D t}}\right)-\frac{2 \sqrt{D t}}{\sqrt{\pi}} \exp \left(-\frac{e^{2}}{4 D t}\right)+\frac{2 \sqrt{D t}}{\sqrt{\pi}}\right]$

Given the values of $e$ and $D$, the terms $\frac{e^{2}}{4 D t}$ and $\frac{2}{2 \sqrt{D t}}$ are very large and therefore all the time that the hypothesis of the semi-infinite medium is valid we can write:

$\bar{X}(x, t)=X_{1}+\left(X_{0}-X_{1}\right) \frac{2 \sqrt{D}}{e \sqrt{\pi}} \sqrt{t}$

The plot of the curve $\bar{X}(x, t)=f(\sqrt{t})$ must have a linear part whose slope $\beta$ makes it possible to estimate the mass diffusivity $D$ by: $D=\frac{\pi \beta^{2} e^{2}}{4\left(X_{0}-X_{1}\right)^{2}}$

Model M2: finite medium, $D$ constant, non-null external resistance, negligible heat transfer We will now develop a M2 model corresponding to the case of a plate of finite thickness $e$ and sealed on the side and rear faces, taking into account the resistance to external mass transfer. 
The boundary conditions are written:

$$
\begin{aligned}
& h_{m}\left[X(x=0)-X_{1}\right]=-\rho_{0} D\left(\frac{\partial X}{\partial x}\right)_{x=0} \\
& \left.\left(\frac{\partial X}{\partial x}\right)_{x=e}=0 \quad \text { (sealed surface in } x=e\right)
\end{aligned}
$$

With these assumptions, the problem is analogous to that of heat transfer in a plate of thickness $2 e$ with heat transfer coefficient imposed on both sides.

The solution can be calculated using several resolution methods leading to solutions of different formulations but all representing the same function, we thus obtain by separation of variables [9]:

$\frac{X(x, t)-X_{1}}{X_{0}-X_{1}}=2 \sum_{n=1}^{\infty} \frac{\sin \left(\omega_{n} e\right)}{\omega_{n} e+s i\left(\omega_{n} e\right) \cos \left(\omega_{n} e\right)} \exp \left(-D \omega_{n}^{2} t\right) \cos \left[\omega_{n}(e-x)\right]$

Where $\omega_{n}(n=1,2, \ldots)$ are the solutions of the equation: $\omega \tan (\omega e)=\frac{h_{m}}{\rho_{0} D}$ that may also be written: $\omega e \tan (\omega e)=\frac{h_{m} e}{\rho_{0} D}$. Thus, the solutions $\omega_{n} e$ of this equation only depend on $\frac{h_{m} e}{\rho_{0} D}$.

This expression can be used to calculate the mean water content $\bar{X}(t)$ of the sample between $x=0$ and $x=e$. We obtain :

$\bar{X}(t)-X_{1}=2\left(X_{0}-X_{1}\right) \sum_{n=1}^{\infty} \frac{\sin ^{2}\left(\omega_{n} e\right)}{\omega_{n} e\left[\omega_{n} e+\sin \left(\omega_{n} e\right) \cos \left(\omega_{n} e\right)\right]} \exp \left(-D \omega_{n}{ }^{2} t\right)$

This relation shows that the mean water content is proportional to $\left(X_{1}-X_{0}\right)$. Otherwise, since the values of $\omega_{n} e$ only depend on $\frac{h_{m} e}{\rho_{0} D}$ and that we have : $D \omega_{n}{ }^{2} t=\frac{D t}{e^{2}}\left(\omega_{n} e\right)^{2}$, one can deduce that $\bar{X}$ only depends on $\frac{h_{m} e}{\rho_{0} D}$ and of the reduced time $t^{*}=\frac{D t}{e^{2}}$. In this case, the following three parameters must be estimated: $X_{0}-X_{1}, D$ and $h_{m}$.

Model M3: Finite medium, $D$ variable, external resistance and heat transfer not neglected

We will assume that there is no liquid diffusion in the solid, which is an acceptable assumption if the relative humidity of the air / product equilibrium is less than $70 \%$. We will further note $D=D_{X}^{v}$. The equation (7) may be rewritten as:

$\rho_{0} \frac{\partial X}{\partial t}=-\frac{\partial}{\partial x}\left[\rho_{0} D\left(\frac{\partial X_{v}}{\partial x}+\delta \frac{\partial T}{\partial x}\right)\right]$

If we assume that there is no liquid diffusion in the material, $D_{X}^{v}$ is given by relation (12) and the coefficient of thermomigration $\delta$ has the expression:

$\delta=\frac{D_{T}^{v}}{D_{X}^{v}}=\frac{p_{s a t} \frac{d H R}{d X}}{H R \frac{d p_{s a t}}{d T}}$

The system of equations to be solved is composed of equations (8) and (31) with the following limiting conditions:

$$
\begin{aligned}
& \left(h_{c}+h_{r}\right)\left[T_{1}-T(x=0)\right]=-\lambda\left(\frac{\partial T}{\partial x}\right)_{x=0} \\
& \left(\frac{\partial T}{\partial x}\right)_{x=e}=0 \\
& h_{v}\left[Y_{1}-Y_{0}\right]=-\rho_{0} D\left(\frac{\partial X}{\partial x}\right)_{x=0} \\
& \left(\frac{\partial X}{\partial x}\right)_{x=e}=0
\end{aligned}
$$

Where the radiation heat transfer coefficient $h_{r}\left(\mathrm{~W} \mathrm{~m}^{-2} \mathrm{~K}^{-1}\right)$ is calculated by (assuming $\varepsilon=$ $0.9)$ :

$h_{r}=4 \sigma \varepsilon T^{3}$

$Y_{1}$ and $Y_{0}$ are the air absolute humidity (expressed in $\mathrm{kg} \mathrm{m}^{-3}$ ) respectively far from the surface and on the surface given by: 
$Y_{1}=\frac{M_{v}}{R T_{1}} p_{v 1}=\frac{M_{v}}{R T_{1}} H R_{1} p_{s a t}\left(T_{1}\right)$

$Y_{0}=\frac{M_{v}}{R T_{(x=0)}} p_{v}(x=0)=\frac{M_{v}}{R T(x=0)} H R(x=0) p_{\text {sat }}[T(x=0)]$

With the approximation : $H R(x=0)=H R_{0}+\left[X(x=0)-X_{0}\right] \frac{d H R}{d X}$

Where $\frac{d H R}{d X}$ is inverse of the slope of the sorption isotherm of the material.

The Chilton-Colburn analogy establishes a relationship between the mass transfer coefficient $h_{v}\left(\mathrm{~m} \mathrm{~s}^{-1}\right)$ and the convective exchange coefficient $h_{c}\left(\mathrm{~W} \mathrm{~m}^{-2} \mathrm{~K}^{-1}\right)[4]$ :

St $\operatorname{Pr}^{\frac{2}{3}}=S t_{m} S c^{\frac{2}{3}}$

Leading to:

$h_{v}=\frac{h_{c}}{\rho c}\left(\frac{D^{*}}{a}\right)^{2 / 3}$

where $a$ and $\rho c$ are respectively the thermal diffusivity and the thermal volume capacity of air. One can simply solve the problem of coupled heat and mass transfer in 1D by an explicit method based on the diagram of figure 2 .

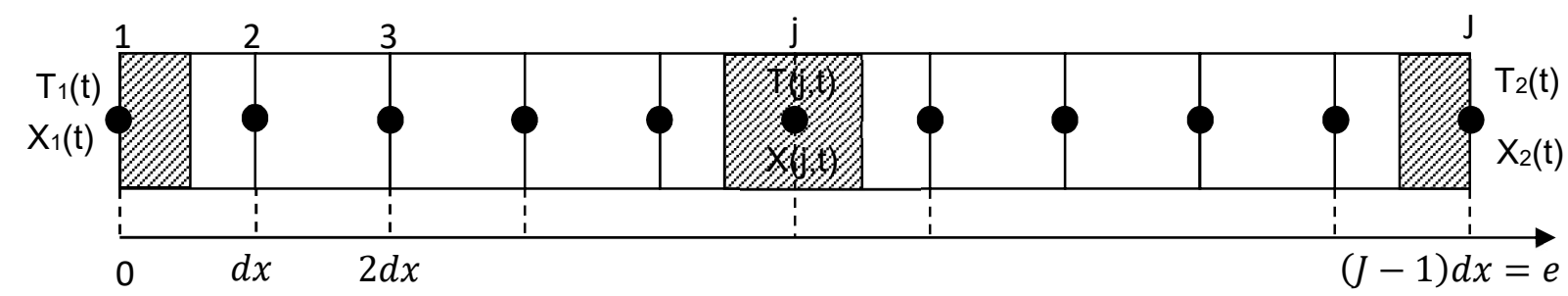

Figure 2: Diagram for numerical resolution

Setting: $T(j, t)=T 1(j) ; T(j, t+d t)=T 2(j) ; X(j, t)=X 1(j)$ and $X(j, t+d t)=X 2(j)$ The heat and mass balances for each type of mesh lead to:

Mesh 1:

$X 2(1)=X 1(1)+\frac{2 d t}{\rho_{0} d x}\left\{h_{v}\left[X_{1}-X 1(1)\right]-\rho_{0} D \frac{X 1(1)-X 1(2)}{d x}-\rho_{0} \delta D \frac{T 1(1)-T 1(2)}{d x}\right\}$
$T 2(1)=T 1(1)+\frac{2}{\rho_{0} c^{*} d x}\left\{h\left[T_{1}-T 1(1)\right] d t-\lambda \frac{T 1(1)-T 1(2)}{d x} d t+\rho_{0} \frac{d x}{2} L[X 2(1)-X 1(1)]\right\}$

(32)

where: $h=h_{c}+h_{r}$

Mesh j:

$X 2(j)=X 1(j)+\frac{D d t}{d x^{2}}\{X 1(j+1)+X 1(j-1)-2 X 1(j)+\delta[T 1(j+1)+T 1(j-1)-$
$2 T 1(j)]\}$
$T 2(j)=T 1(j)+\frac{d t}{\rho_{0} c^{*} d x}\left\{\lambda \frac{T 1(j+1)+T 1(j-1)-2 T 1(j)}{d x}+\rho_{0} L d x \frac{X 2(j)-X 1(j)}{d t}\right\}$

Mesh J:

$X 2(J)=X 1(J)+\frac{2 d t}{d x^{2}}\{-D[X 1(J)-X 1(J-1)]-\delta D[T 1(J)-T 1(J-1)]\}$

$T 2(J)=T 1(J)+\frac{2}{\rho_{0} c^{*} d x}\left\{-\lambda \frac{T 1(J)-T 1(J-1)}{d x} d t+\rho_{0} L \frac{d x}{2}[X 2(J)-X 1(J)]\right\}$

where $L$ is calculated by relation (10).

The simulations have been done using the Matlab code. A sensitivity study of the mesh influence leads us to choose a space interval $d x=\frac{e}{J}$ with $J=60$. The time step was calculated as: 
$d t=\frac{\rho_{0} c^{*}}{\lambda} \frac{d x^{2}}{4}$

Where $c^{*}$ is given by relation (11).

The parameters to be estimated are a priori the coefficient of tortuosity $\tau$, the coefficient of thermomigration $\delta$, the thermal conductivity $\lambda$, the heat and mass transfer coefficients $h$ and $h_{m}$ supposing that $\rho_{0}$ and $c_{S}$ are known.

If we know the sorption isotherm or more simply the inverse of its slope $\frac{d H R}{d X}$, we are brought back to the estimation of 4 unknown parameters: $X_{0}-X_{1}, \tau, \lambda$ et $h_{c}$.

2.3 Validity limits of the models

The hypotheses of a constant diffusivity $D$ and of a negligible effect of the heat transfer on the mass transfer are widely used, the aim of the following study is to investigate their validity domain.

A series of simulations of the evolution of the average water content of the samples was carried out using the coupled heat and mass model M3 with the following values:

$e=10 \mathrm{~mm} ; c_{s}=1000 \mathrm{~J} \mathrm{~kg}^{-1} \mathrm{~K}^{-1} ; \lambda=0.1 \mathrm{~W} \mathrm{~m}^{-1} \mathrm{~K}^{-1} H R_{0}=25 \% ; H R_{1}=60 \%$;

$h_{c}=5 \mathrm{~W} \mathrm{~m}^{-2} \mathrm{~K}^{-1} ; X_{1}-X_{0}=0.01 ; 0.025 ; 0.05 ; T=15^{\circ} \mathrm{C} ; 25{ }^{\circ} \mathrm{C} ; 35^{\circ} \mathrm{C} ; 45^{\circ} \mathrm{C}$

$\rho_{0}=150 \mathrm{~kg} \mathrm{~m}^{-3} ; 300 \mathrm{~kg} \mathrm{~m}^{-3} ; 600 \mathrm{~kg} \mathrm{~m}^{-3} ; \tau=0.01 ; 0.05 ; 0.1 ; 0.25$;

These simulations allow for each simulated case to calculate the evolutions of the water content $X(x, t)$, of the temperature $T(x, t)$ and of the mass diffusion coefficient $D(x, t)$. Figure 3 represents an example of the evolution of the diffusion coefficient $D_{x=0}$ (face exposed) and $D_{x=e}$ (middle of the sample) over time. We can see in this example a strong variation in the diffusion coefficient (about $30 \%$ ) at short times and that at long times this coefficient tends towards a uniform value $D_{\min }$ throughout the sample.

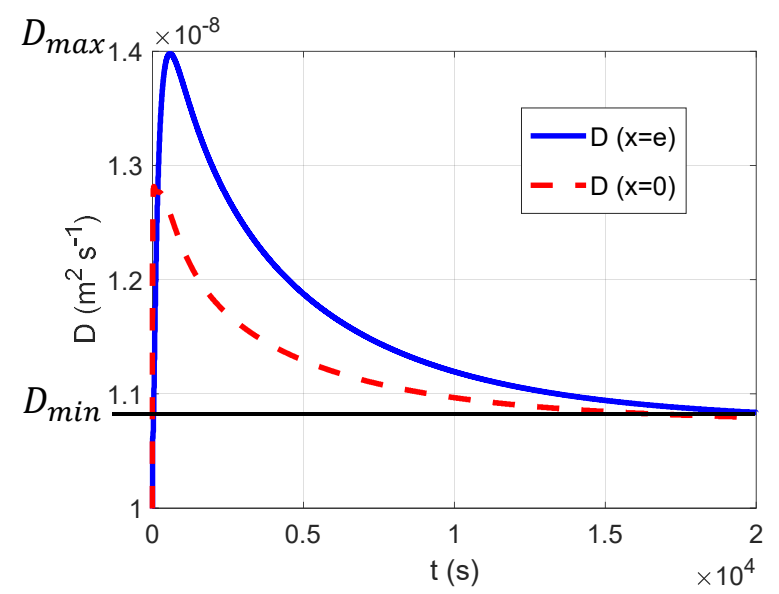

Figure 3: Example of simulated changes in the diffusion coefficient on the external face and in the center of the sample.

The results were then treated as experiments to estimate $D$ and $h$ from the M2 model and it was chosen to calculate the deviations from the values used in the simulations by:

$\Delta D=100 \frac{D-D_{\min }}{D_{\min }}(\%)$

It was found that these deviations did not depend on the density $\rho_{0}$ so all the following simulations were performed with $\rho_{0}=300 \mathrm{~kg} \mathrm{~m}^{-3}$. Figure 4 shows that at fixed tortuosity $\tau$, the estimation error increases when the thermal conductivity decreases. This error also increases very strongly with $\tau$. 


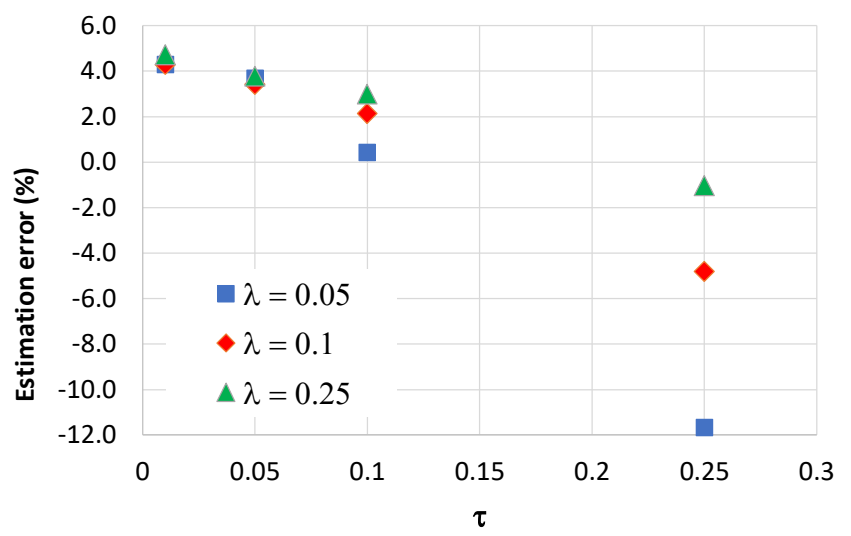

Figure 4: Estimation error at $25^{\circ} \mathrm{C}$ as a function of $\tau$ for different values of $\lambda\left(\mathrm{W} \mathrm{m}^{-1} \mathrm{~K}^{-1}\right)$ with the M2 model $\left(X_{1}-X_{0}=0.03\right)$

Figure 5 shows that the estimation error with model M2 varies very strongly with temperature, which is explained by the exponential form of the mass diffusivity in air $D^{*}$ given by equation (14).

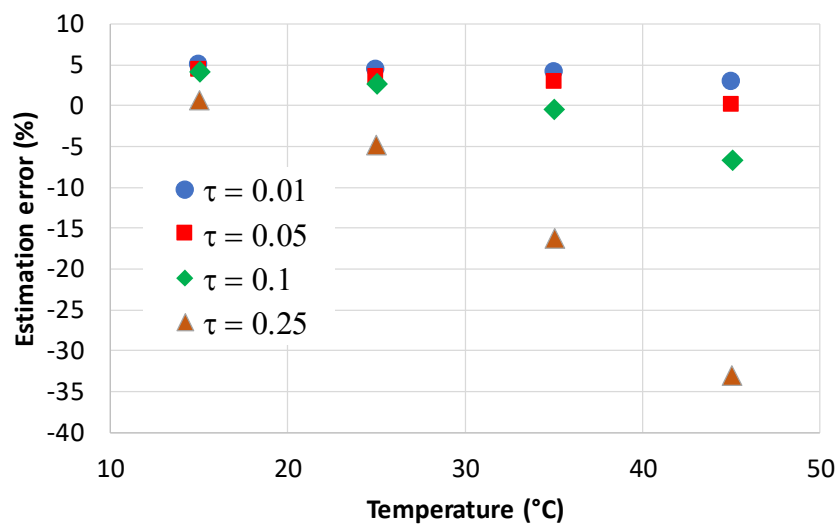

Figure 5: Estimation error as a function of the temperature with the M2 model $(\lambda=$ $0.1 \mathrm{~W} \mathrm{~m}^{-1} \mathrm{~K}^{-1}, \rho_{0}=300 \mathrm{~kg} \mathrm{~m}^{-3}, X_{1}-X_{0}=0.03$ )

Figure 6 shows that when $\tau$ is constant, the estimation error does not vary significantly with $X_{1}-X_{0}$.

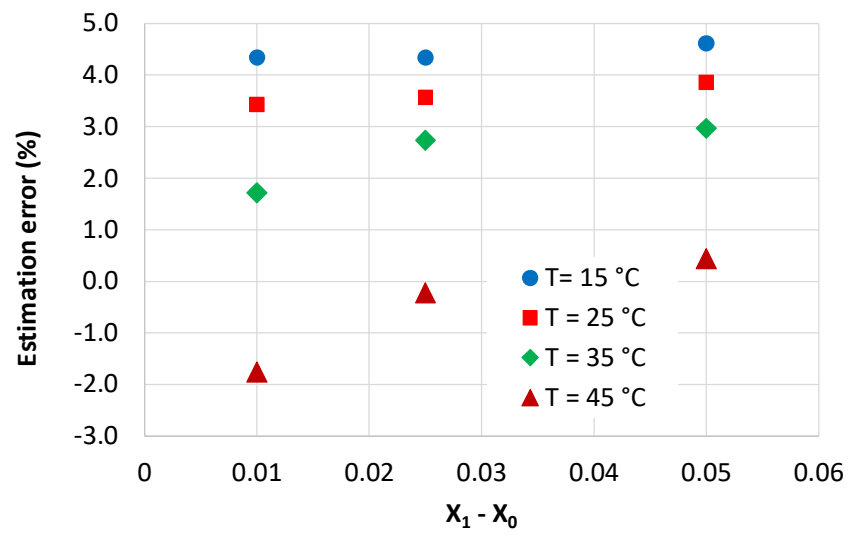

Figure 6: Estimation error as a function of $\left(X_{1}-X_{0}\right)$ with the M2 model $(\lambda=$ $\left.0.1 \mathrm{~W} \mathrm{~m}^{-1} \mathrm{~K}^{-1}, \rho_{0}=300 \mathrm{~kg} \mathrm{~m}^{-3}, \tau=0.05\right)$ 


\section{Sensitivity study}

For each of the two models M2 and M3, the reduced sensitivities of the mean water content to the different parameters were calculated to determine which parameters are estimable and the precision of their estimation. The reduced sensitivity to a parameter $Y$ was calculated numerically by:

$Y \frac{\partial \bar{X}}{\partial Y}=Y \frac{\bar{X}(1,001 Y)-\bar{X}(Y)}{0.001 Y}=1000[\bar{X}(1.001 Y)-\bar{X}(Y)]$

Figures 7 and 8 show the results for models M2 and M3 respectively. The calculations were carried out for the following values of the parameters: $e=10 \mathrm{~mm} ; X_{1}-X_{0}=0.03$;

Model M2: $D=5.83 \times 10^{-9} \mathrm{~m}^{2} \mathrm{~s}^{-1} ; h_{m}=0.005 \mathrm{~kg} \mathrm{~s}^{-1}$

Model M3: $\lambda=0.1 \mathrm{~W} \mathrm{~m}^{-1} \mathrm{~K}^{-1} ; c=1000 \mathrm{~J} \mathrm{~kg}^{-1} \mathrm{~K}^{-1} ; \rho_{0}=300 \mathrm{~kg} \mathrm{~m}^{-3} ; \tau=0.25 ; h_{c}=$ $5 \mathrm{~W} \mathrm{~m}^{-2} \mathrm{~K}^{-1} ; T=25^{\circ} \mathrm{C}$

a)

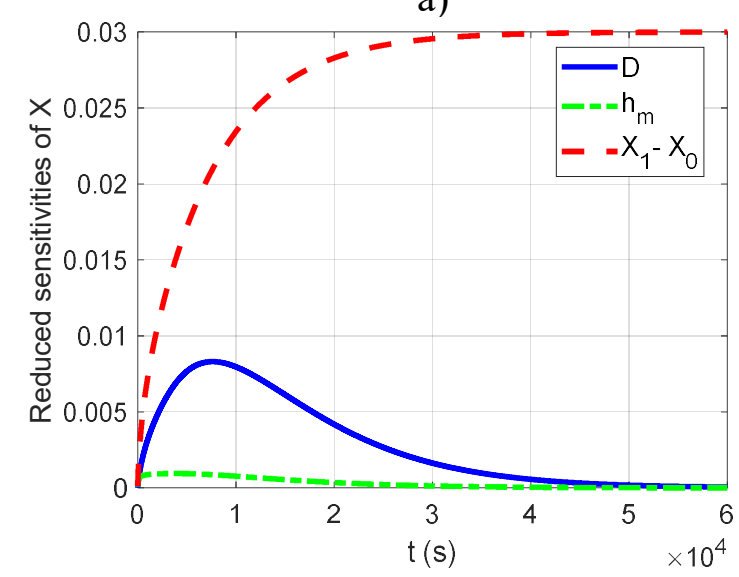

b)

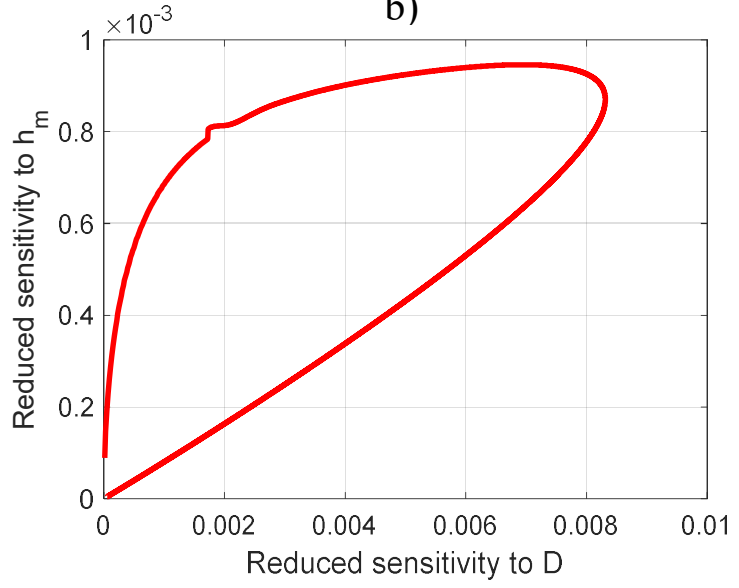

Figure 7: Reduced sensitivities of $\bar{X}(t)$ to the various parameters for model M2.

a)

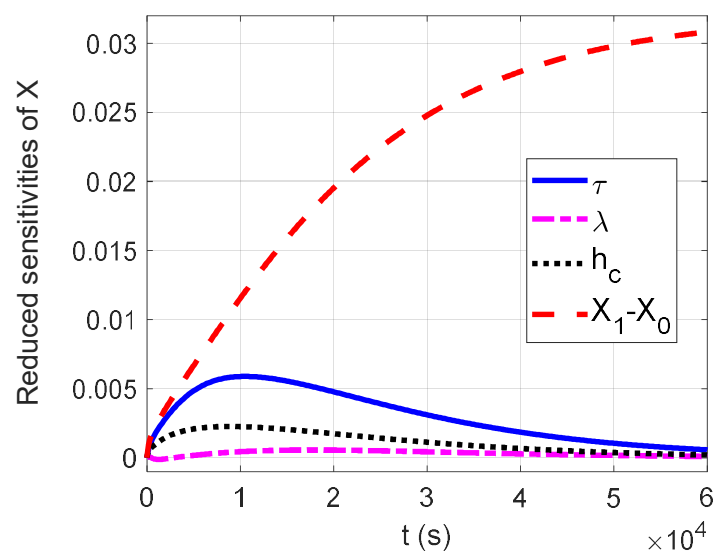

b)

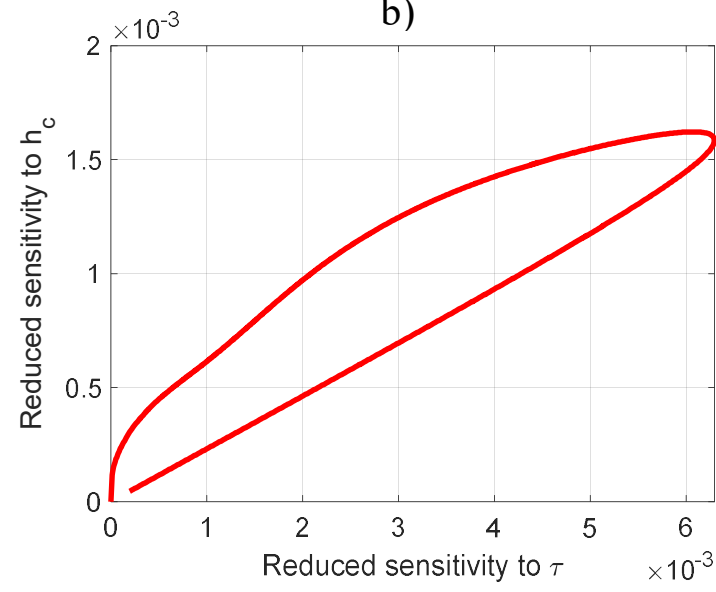

Figure 8: Reduced sensitivities of $\bar{X}(t)$ to the various parameters for model M3.

Figures $7 \mathrm{a})$ and $8 \mathrm{a})$ show that the sensitivity to $\left(X_{1}-X_{0}\right)$ is high and completely uncorrelated with the sensitivities to the other parameters. Therefore, for both the M2 and M3 models, it is not necessary to measure the quantity $\left(X_{1}-X_{0}\right)$ that can be accurately estimated.

Figure 8a) also shows that the sensitivity to $\lambda$ is low. Figure $7 \mathrm{~b}$ ) shows that the sensitivities to $D$ and $h_{m}$ are uncorrelated because the curves representing the sensitivity to $h_{m}$ as a function of the sensitivity to $D$ are not straight lines. For the same reasons, figure $8 \mathrm{~b}$ ) shows that the 
sensitivities to $\tau$ and $h_{c}$ are uncorrelated. These two parameters are therefore estimable separately for the two models M2 and M3.

\section{Material and methods}

The material used is Pavastep, which is a wood fiber board with a density $\rho_{0}=240 \mathrm{~kg} \mathrm{~m}^{-3}$ in dry state, available with a thickness $e=10.85 \mathrm{~mm}$. Its specific heat capacity in the dry state was measured using a SETARAM $\mu \mathrm{dSc} 3$ differential scanning calorimeter and has the value: $c_{s}=1250 \mathrm{~J} \mathrm{~kg}^{-1} \mathrm{~K}^{-1}$.

We used two samples of the same section $10 \times 20 \mathrm{~cm}^{2}$, a thin thermocouple was glued in the center of their interface to assess the influence of mass transfer on temperature. Both samples were then glued together. The side surfaces were finally coated with glue to seal them.

The two glued samples were placed on a Kern BBA-600 balance, taking care to place the exchange surfaces in the direction of the air flow as shown in figure 9. The whole was placed in a Binder KBF 115 climatic chamber which allows to regulate the temperature and relative air humidity.

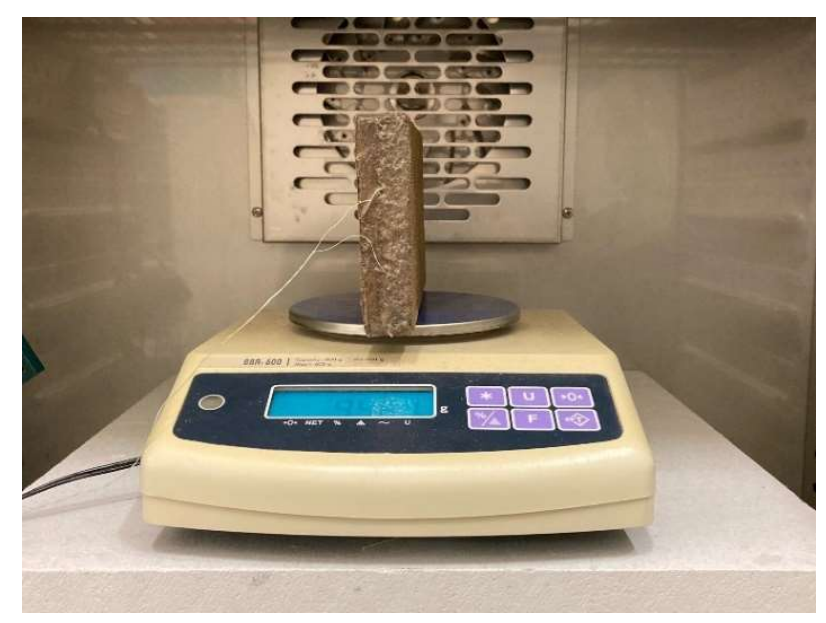

Figure 9: View of the experimental device

The temperature and relative humidity in the chamber were set at $\left(T_{0}, H R_{0}\right)$ and the changes in the mass $m$ of the set of glued samples + thermocouple and of the temperature $T$ at the center of this set were recorded together. Then set the conditions in the oven at $\left(T_{1}, H R_{1}\right)$ and start recording the mass $m$ and the temperature $T$ with a time step of $1 \mathrm{~s}$ until they no longer vary. $\left(X_{1}-X_{0}\right)$ is a parameter estimated by an iterative method, so a value is assigned to it at the start of each iteration.

The mass $m$ is the sum of the dry mass $m_{s}$ of the two samples, of their water mass $m_{w}=X m_{s}$ and of the mass $m_{c}$ of the glue and the thermocouple, we can therefore write:

$m_{0}=\left(1+X_{0}\right) m_{s}+m_{c}$ if the sample is in equilibrium with air at the start of the experiment.

$m=(1+X) m_{s}+m_{c}$

$m_{1}=\left(1+X_{1}\right) m_{s}+m_{c}$ if the sample is in equilibrium with air at the end of the experiment.

$X_{\text {exp }}(t)=X_{0}+\frac{m(t)-m_{0}}{m_{1}-m_{0}}\left(X_{1}-X_{0}\right)$

We assume that $\frac{d H R}{d X}$ is constant and it is calculated by : $\frac{d H R}{d X}=\frac{H R_{1}-H R_{0}}{X_{1}-X_{0}}$. This is equivalent 
assuming that the sorption isotherm is a straight line over the water content interval considered. We then have all the parameters necessary for the computation of $X_{\text {mod }}(t)$. The unknown parameters, namely $\left(X_{1}-X_{0}, D, h_{m}\right)$ for the M2 model or $\left(X_{1}-X_{0}, \tau, \lambda, h_{c}\right)$ for the M3 model, are estimated using the Levenberg-Marquart algorithm to minimize the sum:

$S=\sum_{i=1}^{N}\left[X_{\text {exp }}\left(t_{i}\right)^{2}-X_{\text {mod }}\left(t_{i}\right)^{2}\right]$

where $N$ is the number of experimental points.

For the estimation, we use the "leasqr" function in Matlab. In the version used, the damping parameter changes permanently. At each iteration it is calculated by linear combination of the right singular vectors of the SVD. On the other hand, singular values that are too small are replaced by $10^{-7}$.

\section{Experimental results and discussion}

Measurements were made on the Pavastep samples by fixing the relative humidity of the air in the chamber at $H R_{0}=25 \%$ and $H R_{1}=60 \%$. The measurements were performed at $15{ }^{\circ} \mathrm{C}, 25$ ${ }^{\circ} \mathrm{C}$ and $35{ }^{\circ} \mathrm{C}$ and the values of the mass $m(t)$ and of the temperature $T(e, t)$ were recorded at the center of the sample.

The experimental values of $\bar{X}(t)$ were used to estimate the parameters $X_{1}-X_{0}, D$ and $h_{m}$ from relation (29) (model M2) and the parameters $X_{1}-X_{0}, \tau, \lambda$ and $h_{c}$ from the numerical resolution of the M3 model.

Table 1 shows the results obtained for the three temperatures.

The differences in between the estimates of $D$ presented in Table 1 confirm that, in some cases, the estimate obtained from models M1 and M2 can lead to very large errors. The values estimated by models M1 and M2 are very close. It is also quite remarkable that the values of $\tau$ estimated for the 3 temperatures are very close, we obtain an average value $\tau=0.209$ with a relative standard deviation of $8.1 \%$.

Table 1: Estimation results

\begin{tabular}{|c|c|c|c|c|c|c|c|c|c|}
\hline Model & M1 & \multicolumn{2}{|c|}{$\mathrm{M} 2$} & \multicolumn{5}{c|}{$\mathrm{M} 3$} & \\
\hline$T\left({ }^{\circ} \mathrm{C}\right)$ & $10^{9} \times D$ & $10^{9} \times D$ & $h_{m}$ & $\tau$ & $\lambda$ & $h_{c}$ & $10^{9} \times D_{\min }$ & $10^{9} \times D_{\max }$ & Deviat. \\
\hline${ }^{\circ} \mathrm{C}$ & $\mathrm{m}^{2} \mathrm{~s}^{-1}$ & $\mathrm{~m}^{2} \mathrm{~s}^{-1}$ & & & $\mathrm{~W} \mathrm{~m}^{-2} \mathrm{~K}^{-1}$ & $\mathrm{~W} \mathrm{~m}^{-2} \mathrm{~K}^{-1}$ & $\mathrm{~m}^{2} \mathrm{~s}^{-1}$ & $\mathrm{~m}^{2} \mathrm{~s}^{-1}$ & $\%$ \\
\hline 15 & 3.44 & 3.59 & 0.0018 & 0.229 & 0.093 & 12.0 & 3.58 & 4.02 & -0.3 \\
\hline 25 & 5.34 & 5.01 & 0.0026 & 0.201 & 0.089 & 9.5 & 5.34 & 6.24 & 7.9 \\
\hline 35 & 7.45 & 9.62 & 0.0049 & 0.198 & 0.09 & 10.2 & 11.1 & 13.7 & 13.3 \\
\hline
\end{tabular}

Figure 10 represents the experimental curve, the simulated curve with the estimated parameters and the residuals (difference between the two curves) $\times 10$ obtained with the M2 model. Figure 11 represents the same quantities obtained with the model M3. The M2 model, although leading to an estimation error of more than $13 \%$ gives residuals similar to those obtained with the M3 model. The analysis of the residuals therefore does not provide any information on the validity of the model in this case.

Figure 12 shows the reduced sensitivities calculated using relation (49) of the average water content to the 4 parameters $X_{1}-X_{0}, \tau, \lambda$ and $h_{c}$ of the model M3, calculated with their estimated values at $25{ }^{\circ} \mathrm{C}$. It can be noted that the sensitivity to $X_{1}-X_{0}$ is very high and uncorrelated with other sensitivities so the parameter $X_{1}-X_{0}$ can be estimated with very good accuracy. Sensitivities to the other 3 parameters have time-shifted extrema so they are not correlated and can be estimated separately. The tortuosity $\tau$ is estimated with good accuracy because the higher sensitivity is the one to $\tau$. On the opposite, the sensitivity to thermal 
conductivity is low so this parameter is estimated with low accuracy. The curves also show that the sensitivities to the 3 parameters $\tau, \lambda$ et $h_{c}$ are correlated beyond $30000 \mathrm{~s}$. The estimation will therefore be performed on the time interval $[0 ; 30000 \mathrm{~s}]$.
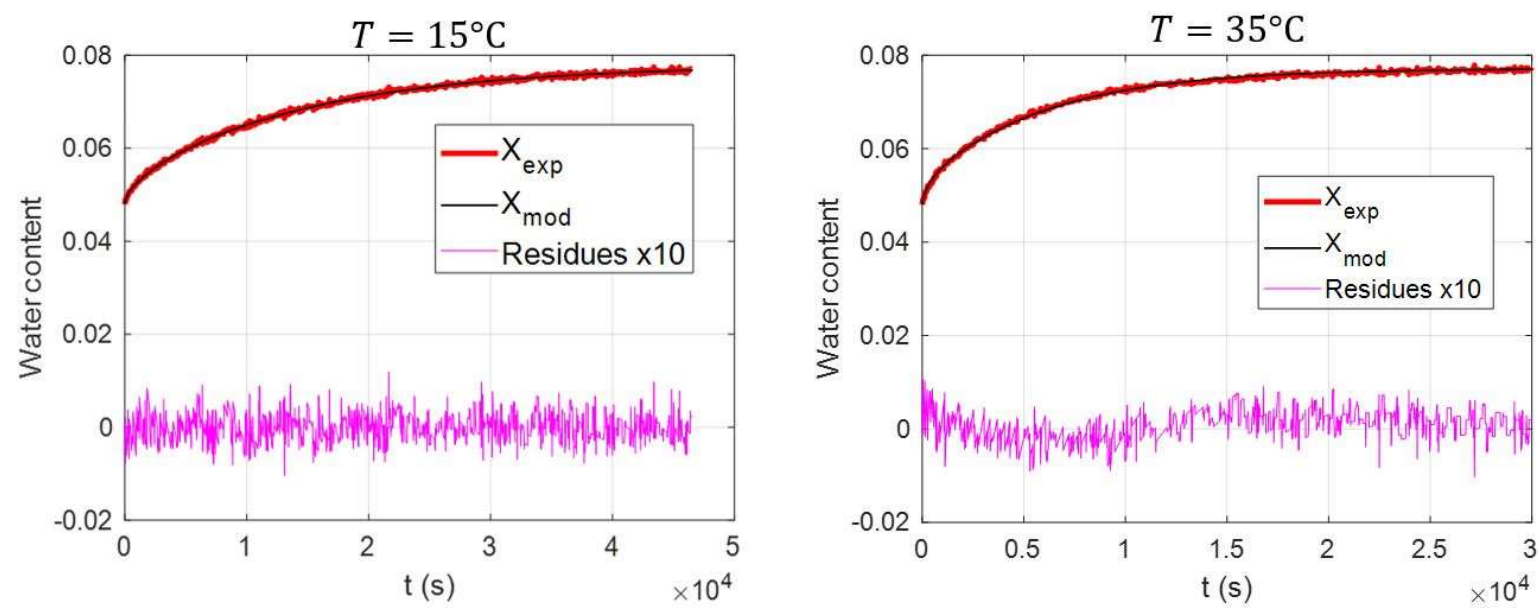

Figure 10: Experimental and simulated curves with estimation residues $\times 10$, obtained with model M2 at $15{ }^{\circ} \mathrm{C}$ and at $35^{\circ} \mathrm{C}$
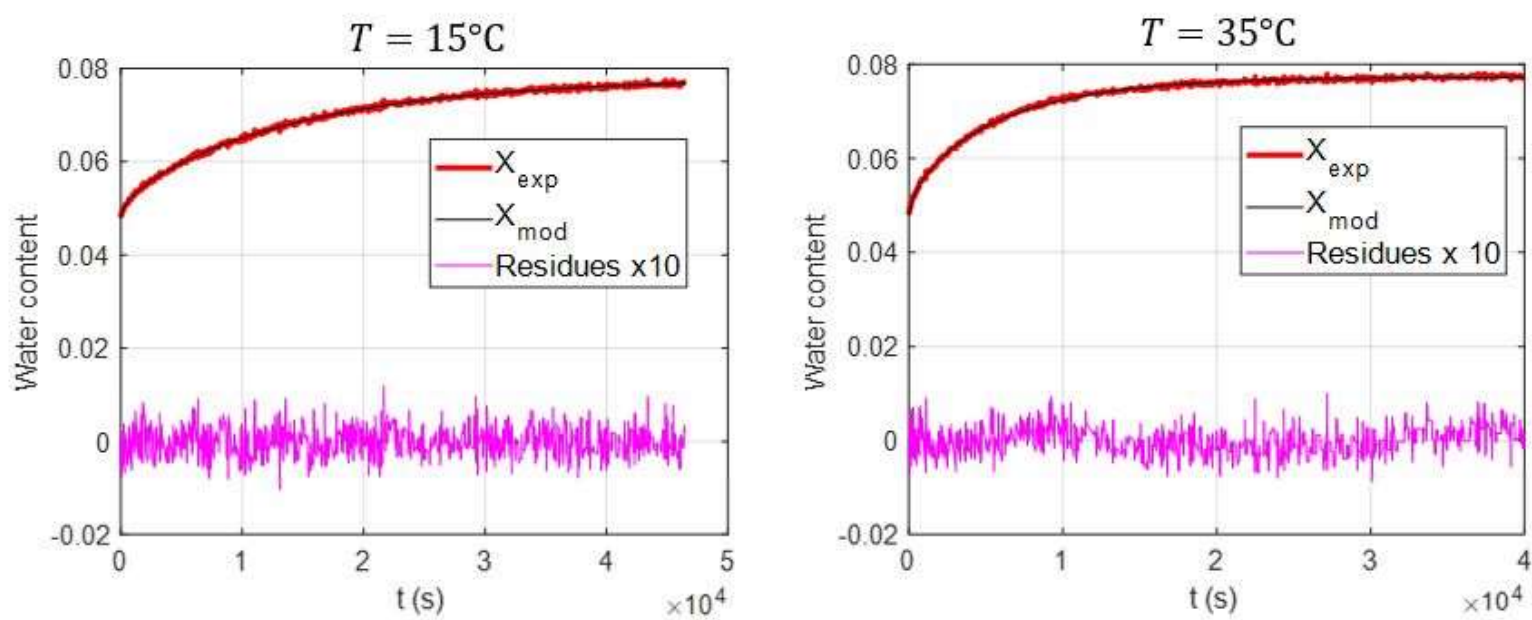

Figure 11: Experimental and simulated curves with estimation residues $\times 10$, obtained with model M3 à $15^{\circ} \mathrm{C}$ et à $35^{\circ} \mathrm{C}$
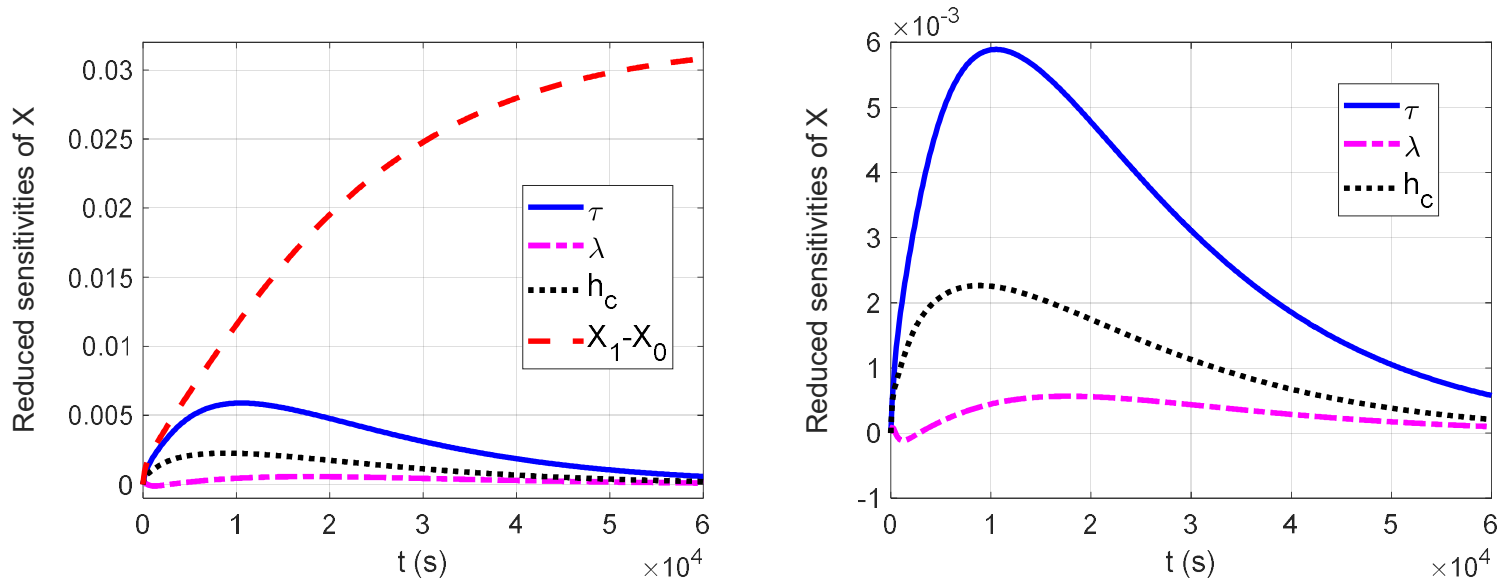

Figure 12: Reduced sensitivities of the mean water content to the parameters $\tau$, $\lambda$ et $h_{c}$ at $25{ }^{\circ} \mathrm{C}$. 
Figure 13 shows the evolution of the simulated diffusion coefficient on the outside face and in the center of the sample. The non-negligible variations of $D$ explain the large estimation errors observed in some cases when $D$ is considered constant.

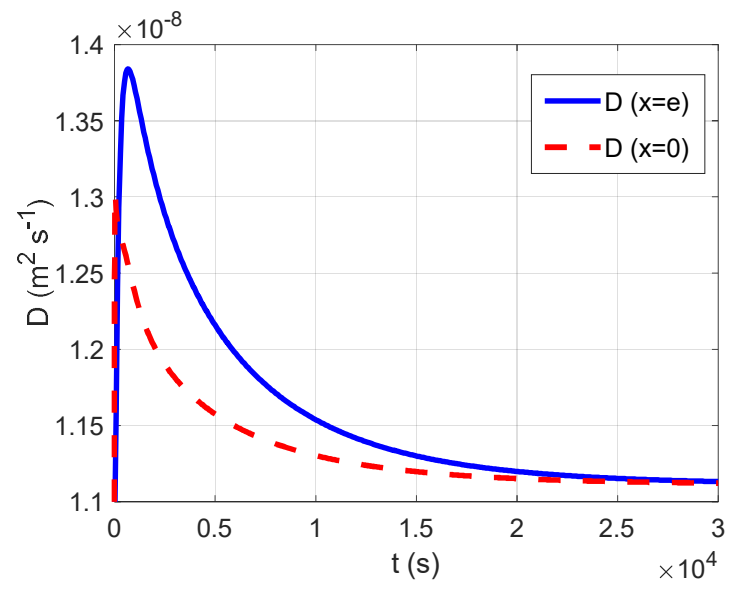

Figure 13: Evolution of the diffusion coefficient on the outside and in the center of the sample $\left(T=35^{\circ} \mathrm{C}\right)$

a)

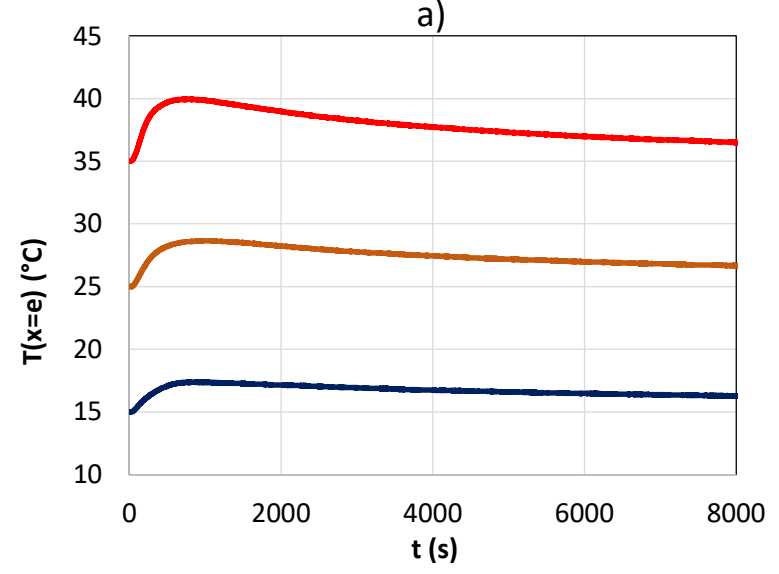

b)

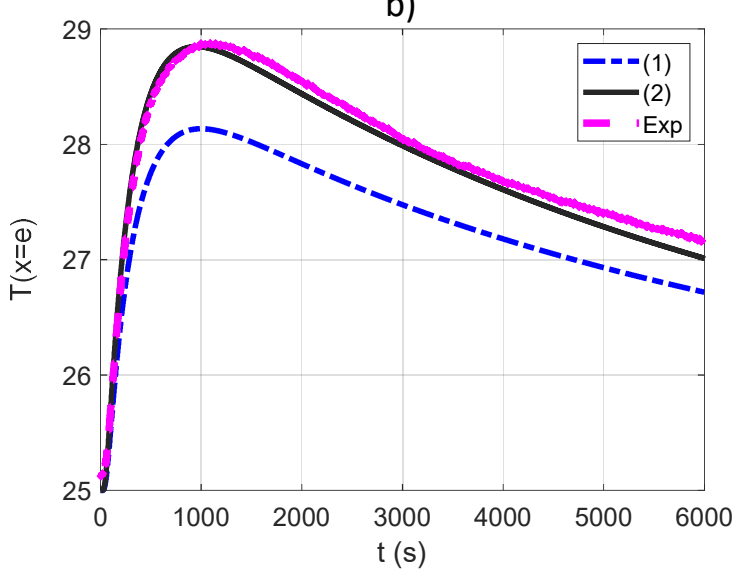

Figure 14: a) Measured temperature at the center of the samples; b) Measured and simulated temperature at $25^{\circ} \mathrm{C}$

Figure 14a) shows the change in temperature measured at the center of the device during the experiments at $15^{\circ} \mathrm{C}, 25^{\circ} \mathrm{C}$ and $35^{\circ} \mathrm{C}$. It can be seen that the higher the temperature, the greater the temperature rise, which can be explained by the increase of the diffusion coefficient with temperature. The rise in temperature due to the mass transfer alone (as $T_{1}=T_{0}$ ) is significant since it can reach $5{ }^{\circ} \mathrm{C}$ in the case where $T_{0}=35^{\circ} \mathrm{C}$. This shows that the mass transfer must be taken into account to calculate the temperature change within this type of material. Figures $14 \mathrm{~b}$ ) represent the experimental temperature curves and first a modelled curve (1) calculated with the values of $X_{1}-X_{0}, \tau$, and $h_{c}$ estimated from the mass measurement at $25^{\circ} \mathrm{C}$. Since the thermal conductivity is not estimated with precision as previously pointed out, its value was fixed to the one given by the manufacturer that is $\lambda=0.05 \mathrm{~W} \mathrm{~m}^{-1} \mathrm{~K}^{-1}$. The shape of the modelled curve (1) is identical to the experimental one but the experimental values are significantly higher than the ones calculated with the model. This can be explained by the fact that the isosteric heat of sorption has been neglected. This hypothesis is confirmed by the modelled curve (2) calculated taking into account the isosteric heat of sorption $\Delta H_{s}$ by replacing 
$L$ by $L+\Delta H_{s}$ in the equations. We considered the following linear form: $\Delta H_{s}=$ $L\left(a+b \frac{X-X_{0}}{X_{1}-X_{0}}\right)$, where $a=0.34$ and $b=-0.32$ have been calculated by regression between $X=0.05$ and $X=0.15$ of the data presented by [9]. The curve (2) is quite close to the experimental one even if the value of the isosteric heat of sorption has not been optimized. This demonstrates that the isosteric heat of sorption has a significant effect on the temperature variation.

It is now necessary to study the influence of the isosteric heat of sorption on the estimation of the diffusion coefficient from mass measurement. This was done with the experimental curves at $15^{\circ} \mathrm{C}, 25^{\circ} \mathrm{C}$ and $35^{\circ} \mathrm{C}$ using the following process:

- A curve was simulated with the values of $X_{1}-X_{0}, \tau, \lambda$ and $h_{c}$ estimated from the mass measurement at respectively $15^{\circ} \mathrm{C}, 25^{\circ} \mathrm{C}$ and $35^{\circ} \mathrm{C}$ (cf. Table 1), but considering $L+\Delta H_{S}$ calculated as previously described instead of $L$,

- This curve was processed as an experimental curve for the parameter estimation considering only the latent heat of vaporization $L$.

It was found that the estimated values of $X_{1}-X_{0}$ and $\tau$ was identical to the values of Table 1 (less than $0.5 \%$ deviation), the latent heat of sorption only influences the estimation of $\lambda$ and $h_{c}$.

One can finally note that the errors in the mass diffusivity estimates reported in Table 1 vary in the same direction as the temperature rise at the center of the sample. A small rise in temperature could therefore be a good criterion for the validity of the M2 model.

\section{Conclusion}

In this paper we propose a coupled heat and mass transfer model with a low number of parameters used for the first time to simulate the evolution of the mass and temperature field in a sample to which a step of humidity is applied to one of its faces. Simulations realized on a wide range of vapor diffusion coefficient, thermal conductivity, density and external mass transfer coefficient have been used to evaluate the error made when using a mass transfer model to estimate the vapor diffusion coefficient from a transient experiment. It has been shown that the estimation error can reach $30 \%$ for low conductivity and highly diffusive materials.

A sensitivity study has shown that a coupled model can be used to estimate separately the vapor diffusion coefficient, the thermal conductivity and the external mass transfer coefficient from a sample's mass recording.

The results of the theoretical study have been confirmed by an experimental study carried on wood fiber boards samples. Estimations of the vapor diffusion coefficient have been estimated both by a mass model and by the proposed coupled model at $15{ }^{\circ} \mathrm{C}, 25{ }^{\circ} \mathrm{C}$ and $35{ }^{\circ} \mathrm{C}$. The deviation between the vapor diffusion coefficient estimated with and without taking into account the heat transfer reached more than $13 \%$ in some cases as predicted by the theoretical study. It is thus necessary to use a coupled model for the estimation of the vapor diffusion coefficient in these cases. It has also been shown that the isosteric heat of sorption (neglected in our model) has no influence on the estimation of the diffusion coefficient but only on the estimation of the thermal conductivity and of the convective external heat transfer coefficient. The measurements of the temperature at the center of the wood fiber boards samples showed that the temperature variation only due to mass transfer can be about $5{ }^{\circ} \mathrm{C}$ so that a coupled heat and mass transfer model should be used to model the temperature field evolution in this type of material. 


\section{References}

1. Agoua E., Zohoun S., Perré P., A double climatic chamber used to measure the diffusion coefficient of water in wood in unsteady-state conditions: determination of the best fitting method by numerical simulation, International Journal of Heat and Mass Transfer 44 (2001) 3731-3744.

2. Azizi, S., Moyne, C., Degiovanni A., Experimental and theoretical approach to thermal conductivity of moist porous media. 1. Experiments, International Journal of Heat and Mass Transfer, 31(11) (1988) 2305-2317.

3. Challansonnex A., Pierre F., Casalinho J., Lv P., Perré P., Mass diffusivity determination of various building materials based on inverse analysis of relative humidity evolution at the back face of a sample, Construction and Building Materials, 193, (2018) 539-546.

4. Chalykh C., Zolotarev P., Chalykh T., Rubtsov A., Zolotova S., Water vapor permeability through porous polymeric membranes with various hydrophilicity as synthetic and natural barriers, Polymers 12 (2020).

5. Chilton T.H., Colburn A.P., Mass transfer (absorption) coefficients prediction from data on heat transfer and fluid friction, Ind. Eng. Chem., 26 (1934) 1183-1187.

6. Chiniforush A. A., Valipour H., Akbarnezahad A., Water vapor diffusivity of engineered wood: effect of temperature and water content, Construction and Buildings Material, 224 (2019) 1040-1055.

7. Gevinga S., Lundea E., Holmeb J., Laboratory Investigations of Moisture Conditions in Wood Frame Walls with Wood Fiber Insulation, Energy Procedia 78 (2015) 1455-1460.

8. Holman J.P., Heat transfer, seventh edition, McGraw-Hill Publishing Company, New York, 1990.

9. Hunter A.J., On the basic equation of sorption and isosteric heat, Wood Science and Technology 25 (1991), 99-111.

10. Jannot Y., L'air humide, 2007, site web http://www.thermique55.com/principal/airhumide.pdf consulté le 13/01/2021.

11. Jannot Y., Moyne C., Transferts thermiques, Edilivre, Paris, 2016.

12. Jannot Y., Degiovanni A., Thermal properties measurement of materials, ISTE \& Wiley, London, 2018.

13. Kaemmerlen A., Asllanaj F., Sallée H., Baillis D., Jeandel G., Transient modeling of combined conduction and radiation in wood fibre insulation and comparison with experimental data, International Journal of Thermal Sciences, 49 (11) (2010) 2169-2176,.

14. Kočíab V., Jermana M., Pavlíka Z., Maděraa J., Žákb J., Černýa R., Interior thermal insulation systems based on wood fiberboards: experimental analysis and computational assessment of hygrothermal and energy performance in the Central European climate, Energy and Buildings 222 (2020).

15. Latif E., Lawrence R.M.H., Shea A.D., Wlaker P., An experimental investigation into the comparative hygrothermal performance of wall panels incorporating wood fibre, mineral wool and hemp-lime, Energy and Buildings, 165 (2018) 76-91.

16. Moyne, C., Batsale J.C., Degiovanni A., Experimental and theoretical approach to thermal conductivity of moist porous media. 2. Theory, International Journal of Heat and Mass Transfer, 31(11) (1988) 2319-2330.

17. Moyne, C., Perré, P., Processes related to drying: Part I, Theoretical Model. Drying Technology, 9 (1991) 1135-1152,. 
18. Nguyen S.T., Tran-Le A.D., Vu M.N., Tob Q.D., Douzane O., Langlet T., Modeling thermal conductivity of hemp insulation material: A multi-scale homogenization approach, Building and Environment, 107 (2016) 127-134.

19. Perré P., Challansonnex A., Colin J ., On the importance of heat and mass transfer coupling for the characterization of hygroscopic insulation materials, International Journal of Heat and Mass Transfer, 133 (2019) 968-975.

20. Raji S., Jannot Y., Lagière P., Puiggali J.R., Thermophysical characterization of a laminated solid-wood pine wall, Construction and Building Materials, 23 (2009) 31893195

21. Rubinoa C., Bonet M., Stefania Liuzzia A.S., Stefanizzia P., Martellottaa F., Wool waste used as sustainable nonwoven for building applications, Journal of Cleaner Production, 278 (2021).

22. Saeidpour M., Wadsö L., Moisture diffusion coefficients of mortars in absorption and desorption, Cement and Concrete Research, 83 (2016) 179-187.

23. Tianyi Zhan, Fengze Sun, Chao Lu, Qian He, Xuan Wang, Kang Xu, Yaoli Zhang, Liping Cai, Evaluation of moisture diffusion in lignocellulosic biomass in steady and unsteady states by a dynamic vapor sorption apparatus, Holzforschung 73 (12), 2019. 